Nortes Martínez-Artero, R. \& Nortes Checa, A. (2017). Competencia matemática, actitud y ansiedad hacia las Matemáticas en futuros maestros. Revista Electrónica Interuniversitaria de Formación del Profesorado, 20 (3), 145-160. DOI: http://dx.doi.org/10.6018/reifop.20.3.290841

\title{
Competencia matemática, actitud y ansiedad hacia las Matemáticas en futuros maestros
}

\author{
Rosa Nortes Martínez-Artero, Andrés Nortes Checa \\ Universidad de Murcia
}

\section{Resumen}

La competencia matemática de los futuros maestros, basada en el dominio de contenidos escolares, es una de las competencias docentes de mayor peso, siendo la actitud y la ansiedad hacia las matemáticas factores importantes en su logro. Para analizar los conocimientos de matemáticas escolares, la actitud y ansiedad hacia las matemáticas, describir la relación entre variables cognitivas y afectivas y señalar errores cometidos, se seleccionaron 174 alumnos de la Universidad de Murcia matriculados el curso 2016/17 a los que se les aplicaron una prueba de matemáticas escolares, un cuestionario de actitud y otro de ansiedad hacia las matemáticas. Los resultados indican que uno de cada tres alumnos del Grado no posee la Competencia Matemática de $6 .^{\circ}$ de Primaria, y que en $2 .^{\circ}$, primer curso con Matemáticas, dos de cada cinco alumnos suspenden la prueba, y uno de cada cinco suspende en los cuatro bloques del currículo de Primaria. Su actitud hacia las matemáticas es ligeramente positiva y no tienen ansiedad ante las matemáticas. Mejores resultados en alumnos que en alumnas, siendo en Geometría donde más errores se cometen. La mitad de los ítems de razonar y reflexionar se suspenden.

\section{Palabras clave}

Competencia matemática; actitud hacia las matemáticas; ansiedad hacia las matemáticas; educación primaria.

\section{Mathematical competence, attitude and anxiety towards mathematics in future Primary school teachers}

\section{Abstract}

Pre-service teachers' mathematical competence, based on a proper command of school curriculum content, is an instrumental teaching competence, and attitude and anxiety are

Contacto:

Rosa Nortes Martínez-Artero, mrosa.nortes@um.es, Facultad de Educación, Universidad de Murcia, Campus de Espinardo, 30100-Espinardo (Murcia). 
important factors when it comes to acquiring this competence. In order to analyse these students' knowledge on school-level mathematics, to know their attitude and anxiety towards mathematics, to describe the relationship between cognitive and affective variables, and to signal frequent mistakes, 174 students from the University of Murcia (academic year 2016/17) were selected and given an exam on school-level mathematics and two questionnaires to measure anxiety and attitude towards mathematics respectively. The results show that one in three students did not have mathematical competence proper of the 6th year of Primary School, and that in the second year (the first year with a subject in mathematics), two in five students failed the test and one in five students failed all the blocks established in the Primary School Official Curriculum. Their attitudes towards mathematics was slightly positive and they did not have anxiety to mathematics. Men obtained better results than women and it was in Geometry that more mistakes were made. Students failed half the items implying reasoning and reflecting.

\section{Key words}

Mathematical competence; attittude to mathematics; anxiety to mathematics; Primary School Education.

\section{Introducción}

Una de las competencias docentes con más peso en el Grado de Maestro de Primaria tiene que ver con el dominio de contenidos escolares (Martín del Pozo, Fernández-Lozano, González-Ballesteros y de Juanas, 2013).

Las matemáticas escolares son las matemáticas consideradas como objeto de enseñanza y aprendizaje (Rico, Marín, Lupiáñez y Gómez, 2008) y forman parte de los conocimientos especializados propios de la profesión de maestro, ya que en la Orden ECI/3857/2007 por la que se establecen los requisitos para la verificación de los títulos universitarios oficiales que habilitan para el ejercicio de la profesión de Maestro en Educación Primaria, en el módulo didáctico disciplinar correspondiente a la materia Enseñanza y Aprendizaje de las Matemáticas, se establece como competencias que deben adquirirse "conocer el currículo escolar de matemáticas" (p. 53750).

\section{Antecedentes}

El alumno que accede al Grado de Maestro de Primaria (GMP) trae unos conocimientos de matemática escolar basados en conocimientos conceptuales y procedimentales y que en el transcurso de sus estudios "debe conocer, entender y utilizar aquellas nociones de matemáticas que ha de enseñar y transmitir a sus futuros alumnos de Primaria, con el nivel de reflexión y la amplitud de análisis requeridos para desenvolverse con soltura en una clase de Primaria" (Segovia y Rico, 2011, p. 21).

¿Cómo saber los conocimientos de matemáticas escolares que traen los alumnos que acceden al Grado de Maestro de Primaria? "El bagaje matemático previo del estudiante que comienza en primero de universidad es una variable importante a la hora de comprender el fenómeno de dificultades de origen afectivo en matemáticas" (Gómez-Chacón, 2009, p. 28), ya que una mejor preparación conlleva una menor reacción de estrés ante una situación de resolución de problemas. 
Castro, Mengual, Prat, Albarracín y Gorgorió (2014) establecen el concepto de "Conocimiento Matemático Fundamental (CMF) como aquél conocimiento disciplinar en matemáticas necesario para seguir con aprovechamiento las materias de Matemáticas y Didáctica de las Matemáticas del Grado en Educación Primaria” (p. 227). Pero, ¿cuál debe ser ese conocimiento disciplinar?

Como no existe todavía un acuerdo generalizado, se acude a pruebas de distinta índole cuyo contenido son las matemáticas escolares de Primaria. Pruebas del tipo de Conocimientos y destrezas indispensables (CDI) para $6 .^{\circ}$ de Primaria o Pruebas para el procedimiento selectivo para el ingreso en el cuerpo de maestros de la Comunidad de Madrid han sido empleadas en anteriores investigaciones (Nortes, 2011; Nortes y Nortes, 2016) con la finalidad de conocer el bagaje de matemáticas elementales de los alumnos que acceden al Grado de Maestro de Primaria.

Lacasa y Rodríguez (2013) en el informe español del Estudio internacional sobre la formación inicial en Matemáticas de los maestros obtienen un resultado importante y es la correlación entre el nivel de conocimientos matemáticos y su nivel de conocimientos de didáctica de las matemáticas $(r=0,38)$, argumentando que quienes acaban dominando mejor la didáctica de las matemáticas son aquellos que mejor dominan las matemáticas y "habría que apostar por una selección rigurosa de los candidatos a las carreras de Maestro en términos de conocimientos matemáticos" (p. 83).

En Cataluña a partir del curso 2017/18, los estudiantes que acceden al Grado de Maestro de Primaria e Infantil tienen que superar, entre otras pruebas, las que evalúan las competencias lógico-matemáticas, resolviendo, sin calculadora, ejercicios, problemas y situaciones de aplicación, teniendo que poner en juego conocimiento conceptual y procedimental. Porque uno de los principales motivos de los errores de los alumnos es la falta de comprensión de los contenidos matemáticos, que acaban memorizando procedimientos y hechos, razón por la cual los alumnos tienen dificultades para argumentar las decisiones que toman cuando resuelven problemas (Pañellas, 2016).

Martín del Pozo et al. (2013) a una muestra de 343 maestros de 85 centros de la Comunidad de Madrid que tenían un acuerdo con la Facultad de Educación de la Universidad Complutense de Madrid para que los estudiantes de Magisterio realizaran allí sus prácticas docentes les plantearon la siguiente cuestión ¿Qué valoración hacen los maestros en activo del dominio de los contenidos escolares como competencia profesional? De las 28 competencias propuestas en el cuestionario, el dominio de los contenidos escolares es la tercera más valorada en cuanto a su importancia para la profesión y en cuanto a su utilización en la práctica docente, siendo la implicación de las familias en el aprendizaje de los hijos y la educación en valores las que ocupan los primeros puestos.

El artículo 12 del Real Decreto 126/2014 correspondiente a evaluaciones indica que "al finalizar el sexto curso de Educación Primaria se realizará una evaluación final individualizada a todos los alumnos y alumnas en la que se comprobará el grado de adquisición de la competencia en Comunicación lingüística, de la Competencia matemática..." (p. 19358). De ahí, que la Prueba de Competencia Matemática de 6. curso sea considerada válida como prueba externa para conocer los conocimientos de matemáticas escolares de los alumnos que acceden al GMP.

Si importante es conocer el nivel de matemáticas escolares, no menos importante es el conocimiento de la actitud y la ansiedad que tienen hacia las Matemáticas. Al GMP acceden alumnos con una serie de conocimientos distintos de matemáticas escolares y de actitud hacia las matemáticas. La confianza que tenga un alumno en sus propias capacidades y posibilidades influye de manera significativa sobre los resultados que alcanza en una prueba 
de matemáticas, de tal forma que los resultados parecen sugerir que una actitud positiva o negativa depende en cierta manera de la seguridad o inseguridad que se tenga hacia las matemáticas (estrada y Díaz-Palomar, 2011).

A la falta de comprensión de los contenidos matemáticos elementales, que genera en los estudiantes una falta de confianza y de seguridad en ellos mismos, se une la actitud que se tenga hacia las Matemáticas y la ansiedad que se genera ante la materia sobre todo cuando se resuelven problemas.

Una de las principales preocupaciones en la Educación Matemática es la investigación en torno a los errores en el proceso de aprendizaje. Abrate, Pochulu y Vargas (2006) en una investigación sobre errores y dificultades en matemáticas hacen un estudio con 273 estudiantes aspirantes a ingresar en las Carreras de Ciencias Económicas que cursaban el Módulo de Matemáticas del Curso de Ingreso, a los que aplicaron 8 ejercicios con subapartados y en donde la ausencia de respuestas fue del $21 \%$ de la evaluación total. Los errores cometidos más destacados son: errores debidos a inferencias o asociaciones incorrectas $(27,43 \%)$, errores debidos a dificultades para obtener información espacial $(24,28 \%)$ y errores debidos a ausencia de conocimientos previos (20,73\%).

Alguacil, Boqué y Pañellas (2016) analizan los errores cometidos por 226 estudiantes de $3 .^{\circ} \mathrm{y}$ $4 .^{\circ}$ del GMP utilizando dos pruebas de conocimientos básicos en Matemáticas llegando a establecer una primera tabla de errores básicos más frecuentes en numeración y cálculo y una segunda de errores básicos más frecuentes en medida, estableciendo en cada una contenido, concepto en el que muestran la dificultad, ejemplo del error y carencias que evidencian.

En Nortes y Nortes (2016) se aplicó a una muestra de 142 alumnos del GMP la prueba de Matemáticas para el Ingreso en el Cuerpo de Maestro de Primaria de 2013 de la Comunidad de Madrid, de contenidos correspondientes a $6 .^{\circ}$ de Primaria, que consta de 15 cuestiones y en donde la mitad de los alumnos contestó bien la tercera parte de las cuestiones de la prueba, llegando el porcentaje de error al $80 \%$ en tres de las cuestiones.

La competencia matemática, la ansiedad ante las matemáticas, la actitud hacia las matemáticas y el análisis de errores forman parte de la enseñanza-aprendizaje de las matemáticas en los futuros maestros de Primaria y de su profesionalización, de ahí que se intente conocer la situación actual para planificar la formación inicial de los estudios para maestro de Primaria.

\section{Objetivos}

Los objetivos de la presente investigación son:

1. Analizar los conocimientos de matemática escolar de los alumnos del Grado de Maestro de Primaria por bloques de contenidos, curso y sexo.

2. Analizar la actitud y la ansiedad hacia las matemáticas de los futuros maestros.

3. Describir la relación que existe entre las variables de dominio cognitivo y afectivo.

4. Describir errores cometidos por bloques de contenido y por sexo. 


\section{Metodología}

\section{Participantes}

Fueron 174 alumnos matriculados el curso 2016/17 en el Grado de Maestro de Primaria de la Universidad de Murcia, 59 de segundo, 75 de tercero y 40 de cuarto, cursos en donde se imparte una asignatura de la materia enseñanza-aprendizaje de las Matemáticas. De edades comprendidas entre 18 y 48 años y edad media 21,275 años. El 30,5\% eran hombres y el 69,5 $\%$ eran mujeres. Los alumnos elegidos fueron los asistentes a clase el día que se aplicaron las pruebas.

\section{Diseño}

De corte descriptivo tipo encuesta. Se trata de una investigación cuantitativa de tipo descriptivo encaminada a caracterizar una situación concreta, presentando tablas con la información resumida y analizando posteriormente los resultados con la finalidad de extraer generalizaciones significativas que contribuyan al conocimiento de competencia matemática, actitud y ansiedad hacia las matemáticas en futuros maestros.

\section{Instrumento}

1. Prueba de evaluación final de Educación Primaria. Competencia Matemática $6 .^{\circ}$ curso de Educación Primaria. Curso 2015-2016 (INEE, 2016). Consta de 35 cuestiones correspondientes a $6 .^{\circ}$ de Primaria de los bloques números, medida, geometría e incertidumbre, con preguntas de respuesta cerrada y semiestructurada y de distintos grados de dificultad. En esta prueba hay que responder a preguntas relacionadas con distintas situaciones. La fiabilidad obtenida es: Alfa de Cronbach $=.875$.

2. Cuestionario de Actitud hacia las Matemáticas de Auzmendi (1992). Consta de 25 frases en donde hay que indicar si se está totalmente en desacuerdo (TD), en desacuerdo (D), ni en desacuerdo ni acuerdo ( $\mathrm{N}$ ), de acuerdo (A) o totalmente de acuerdo (TA). Hay frases escritas de forma positiva y otras de forma negativa. La escala es tipo Likert de 1 a 5 . La fiabilidad obtenida es: Alfa de Cronbach $=.871$.

3. Cuestionario de Ansiedad hacia las Matemáticas de Fennema y Sherman (1976). Consta de 12 frases en donde hay que indicar de 1 a 5 si se está totalmente en desacuerdo hasta si se está totalmente de acuerdo. Hay frases en positivo y en negativo. La fiabilidad obtenida es: Alfa de Cronbach $=.879$.

En la Prueba de Competencia Matemática (INEE, 2016), el Proceso Cognitivo y la Dificultad de los bloques es la siguiente:

- Números: 3 ítems de Razonar y Reflexionar (RR), 3 de Aplicar y Analizar (AA) y 5 de Conocer y Reproducir (CR). Hay 5 ítems de dificultad Baja (B), 4 de dificultad Media (M) y 2 de dificultad Alta (A).

- Medida: 2 de RR, 6 de AA y 1 de CR. Hay 2(B), $5(M)$ y $2(A)$.

- Geometría: 3 de RR, 4 de AA y 1 de CR. Hay $1(B)$ y $7(M)$.

- Incertidumbre: 2 de RR, 2 de $A A$ y 3 de $C R$. Hay 2(B), 2(M) y $3(A)$.

Para la puntuación de la Prueba se han considerado las 35 preguntas bien contestadas como 10 y en cada bloque la puntuación total como 10, siendo 11 ítems en Números, 9 ítems en Medida, 8 ítems en Geometría y 7 ítems en Incertidumbre.

En las escalas de Actitud y Ansiedad, medidas de 1 a 5, el valor 3 equivale a "ni en desacuerdo ni acuerdo", considerando una actitud positiva por encima de 3 y negativa por debajo de 3 y en ansiedad a la inversa. 


\section{Procedimiento}

Las pruebas se aplicaron la primera semana de clase con la finalidad de conocer como llegaban a $2 .^{\circ}$ (primer curso con asignaturas de Matemáticas) los alumnos que accedían al Grado de Maestro de Primaria (GMP), cómo empezaban $3 .^{\circ}$ después de haber cursado 12 créditos de Matemáticas y su didáctica, y cómo se encontraban en $4 .^{\circ}$, último curso del Grado, después de haber cursado 21 créditos de Matemáticas y su didáctica. Los tres cuestionarios se pasaron conjuntamente teniendo un tiempo limitado para su contestación. Los resultados se obtuvieron utilizando el paquete estadístico Systat 13.0.

\section{Resultados}

\section{Por bloques de contenidos, curso y sexo}

Para dar respuesta a los dos primeros objetivos que se centraron en analizar los conocimientos de matemática escolar de los alumnos del Grado de Maestro de Primaria por bloques de contenidos, curso y sexo y en analizar la actitud y la ansiedad hacia las matemáticas de los futuros maestros, se presentan tablas con resultados por bloque de contenidos, de actitud y ansiedad, tanto de medias como de porcentajes, analizando su contenido.

En la tabla 1 se presentan los resultados por bloque: Números (NUM), Medida (MED), Geometría (GEO) e Incertidumbre (INC), total de la Prueba de Competencia Matemática (PCM), Actitud (ACT) y Ansiedad (ANS).

Tabla 1.

Resultados de la prueba por bloques, actitud y ansiedad

\begin{tabular}{llllllll}
\hline & NUM & MED & GEO & INC & PCM & ACT & ANS \\
\hline $\mathrm{N}$ & 174 & 174 & 174 & 174 & 174 & 174 & 174 \\
Mínimo & 0 & 1.110 & 0 & 1.430 & 1.140 & 2.120 & 1.250 \\
Máximo & 10 & 10 & 10 & 10 & 9.710 & 4.480 & 4.583 \\
Media & 6.159 & 5.754 & 5.295 & 6.666 & 5.967 & 3.223 & 2.985 \\
D. T. & 2.189 & 2.174 & 2.734 & 2.216 & 2.000 & 0.497 & 0.720 \\
\hline
\end{tabular}

- En ningún bloque se alcanza una media de Notable.

- Hay 10 alumnos con sobresaliente. Por curso, 1 es de $2 .^{\circ}, 5$ son de $3 .^{\circ}$ y 4 son de $4 .^{\circ}$. Por sexo son 4 hombres y 6 mujeres.

- Hay 15 alumnos con puntuación inferior a 3 . Por curso 10 son de $2 .^{\circ}, 3$ son de $3 .^{\circ}$ y 2 son de $4 .^{\circ}$. Por sexo 1 hombre y 14 mujeres.

- Hay 59 alumnos que suspenden. Por curso, 26 son de $2 .^{\circ}, 19$ son de $3 .^{\circ}$ y 14 son de $4 .{ }^{\circ}$. Por sexo son 11 hombres y 48 mujeres.

- Hay 16 alumnos con ansiedad mayor e igual a 4. Por curso, 2 son de $2 .^{\circ}, 10$ son de $3 .^{\circ}$ y 4 son de $4 .^{\circ}$. Por sexo son 5 hombres y 11 mujeres.

- Ningún alumno tiene actitud igual o inferior a 2.

- En los resultados por ítems hay 9 sin aprobar que corresponden 5 a Razonar y Reflexionar, 3 a Aplicar y Analizar y 1 a Conocer y Reproducir. 
En la tabla 2 se presentan porcentajes de suspensos por curso y por sexo en cada uno de los bloques.

Tabla 2.

Porcentajes de suspensos de bloques, por curso y sexo

\begin{tabular}{lllllll}
\hline SUSPEN & $\mathbf{2 .}^{\circ}$ & $3^{\circ}$ & $\mathbf{4 .}^{\circ}$ & TOTAL & HOMBRE & MUJER \\
\hline NUM & 35.59 & 22.67 & 30.00 & 31.03 & 24.42 & 33.06 \\
MED & 44.07 & 33.33 & 30.00 & 36.21 & 20.75 & 42.98 \\
GEO & 50.85 & 29.33 & 40.00 & 39.08 & 26.42 & 44.63 \\
INC & 35.59 & 17.33 & 22.50 & 24.71 & 9.43 & 31.40 \\
PCM & 44.07 & 25.33 & 35,0 & 33.91 & 22.64 & 37.19 \\
\hline
\end{tabular}

- Uno de cada tres futuros maestros no tiene adquirida la Competencia Matemática de $6 .^{\circ}$ de Primaria.

- Geometría es el bloque con peores resultados, siendo los alumnos de $2 .^{\circ}$ los que más suspenden, uno de cada dos.

- En todos los bloques los alumnos suspenden menos que las alumnas.

- Es en $3 .^{\circ}$ donde se obtienen mejores resultados, suspende uno de cada cuatro alumnos.

Se presentan en la tabla 3 los porcentajes de alumnos por curso y sexo que tiene 4, 3, 2, 10 o bloques aprobados.

Tabla 3.

Resultados de bloques aprobados, por curso y sexo, en porcentaje

\begin{tabular}{lllllll}
\hline APROB & $\mathbf{2 .}^{\circ}$ & $3^{\circ}$ & $\mathbf{4 .}^{\circ}$ & TOTAL & HOMBRE & MUJER \\
\hline 4 BL. & 37.29 & 49.33 & 52.5 & 45.98 & 60.38 & 39.67 \\
3 BL. & 18.64 & 21.33 & 10.00 & 17.82 & 18.87 & 17.36 \\
2 BL. & 8.47 & 9.33 & 10.00 & 9.20 & 5.66 & 10.74 \\
1 BL. & 13.60 & 13.33 & 1750 & 14.37 & 11.32 & 15.70 \\
O BL. & 22.03 & 6.67 & 10.00 & 12.64 & 3.77 & 16.53 \\
N & 59 & 75 & 40 & 174 & 53 & 121 \\
\hline
\end{tabular}

- En $2 .^{\circ}$ uno de cada cinco estudiantes suspende en todos los bloques.

- En mujeres cuatro de veinticinco alumnas no aprueba ningún bloque.

- Solo en $4 .^{\circ}$ uno de cada dos estudiantes aprueba los cuatro bloques.

- Tres de cada cinco hombres aprueban los cuatro bloques, mientras que en mujeres lo son dos.

Se aplica la t de Student para conocer si hay diferencias significativas en cada uno de los bloques, en el total, en actitud y en ansiedad. Se presentan los resultados en la tabla 4. 
Tabla 4.

Resultados por sexo

\begin{tabular}{llllllll}
\hline & NUM & MED & GEO & INC & PCM & ACT & ANS \\
\hline HOM & 6.466 & 6.458 & 6.085 & 7.438 & 6.610 & 3.259 & 2.840 \\
MUJ & 6.025 & 5.446 & 4.948 & 6.328 & 5.686 & 3.208 & 3.048 \\
$t$ & 1.224 & 2.887 & 2.565 & 3.117 & 2.862 & 0.626 & -1.768 \\
$p$ & .222 & .004 & .011 & .002 & .005 & .532 & .079 \\
\hline
\end{tabular}

- En todas las variables mejores resultados en hombres que en mujeres.

- Hay diferencias significativas en Medida, Geometría, Incertidumbre y Prueba.

- $\quad \mathrm{Ni}$ en Números, Actitud y Ansiedad las diferencias son significativas por sexo.

Se aplica una F de Snédecor para conocer en cada bloque, Prueba, Actitud y Ansiedad si hay diferencias significativas. Se presenta la tabla 5 con los resultados.

Tabla 5.

Resultados por curso

\begin{tabular}{llllllll}
\hline & NUM & MED & GEO & INC & PCM & ACT & ANS \\
\hline $2 .^{\circ}$ & 5.731 & 5.330 & 4.195 & 6.101 & 5.351 & 3.247 & 2.856 \\
$3 .^{\circ}$ & 6.533 & 5.941 & 5.883 & 7.104 & 6.324 & 3.178 & 3.108 \\
$4 .^{\circ}$ & 6.090 & 6.029 & 5.813 & 6.678 & 6.207 & 3.274 & 2.944 \\
$F$ & 2.273 & 1.735 & 7.800 & 3.475 & 4.450 & 0.589 & 2.218 \\
$p$ & .106 & .179 & .001 & .033 & .013 & .556 & .122 \\
\hline
\end{tabular}

- En $2 .^{\circ}$ se obtienen las medias más bajas en todos los bloques.

- En $3 .^{\circ}$ se obtienen las medias más altas en tres bloques, no en medida.

- La mejor actitud hacia las matemáticas la tienen los alumnos de $4 .^{\circ}$.

- La menor ansiedad la tienen los alumnos de 2.․

- En Números y Medida no hay diferencias significativas por curso.

- En Geometría ( $p=.001)$ e Incertidumbre $(p=.033)$ hay diferencias significativas en detrimento de $2 .^{\circ}$.

- En el conjunto de la prueba hay diferencias significativas $(p=.013)$ en detrimento de 2. ${ }^{\circ}$ con la puntuación más baja.

- No hay diferencias significativas por curso ni en actitud ni en ansiedad. En $3 .^{\circ}$ la actitud es más baja y la ansiedad más alta que en $2 .^{\circ}$ y $4 .^{\circ}$.

Correlaciones entre variables de dominio cognitivo y afectivo

Para dar respuesta al tercer objetivo que se centró en describir la relación que existe entre las variables de dominio cognitivo y afectivo, se presenta la tabla 6 con las correlaciones entre las variables consideradas, tanto de dominio cognitivo como afectivo. 
Tabla 6.

Correlaciones entre variables

\begin{tabular}{llllllll}
\hline & NUM & MED & GEO & INC & PCM & ACT & ANS \\
\hline NUM & 1.000 & & & & & & \\
MED & .664 & 1.000 & & & & & \\
GEO & .664 & .694 & 1.000 & & & & \\
INC & .666 & .583 & .631 & 1.000 & & & \\
PCM & .878 & .857 & .869 & .805 & 1.000 & & \\
ACT & .142 & .152 & .246 & .117 & .202 & 1.000 & \\
ANS & -.240 & -.196 & -.296 & -.164 & -.265 & -.715 & 1.000 \\
\hline
\end{tabular}

- Las correlaciones entre bloques de contenidos son altas y significativas.

- Las correlaciones entre cada bloque y el total (PCM) son superiores a .8.

- Actitud y Ansiedad correlacionan inversamente con un valor alto.

- Actitud y Prueba correlacionan con un valor bajo, que no llega a ser significativo $(p=.159)$.

- Ansiedad y Prueba (PCM) correlacionan negativamente con un valor bajo, pero significativo $(p=.009)$.

\section{Errores}

Para dar respuesta al cuarto objetivo centrado en describir errores cometidos por bloques de contenido, se calculan porcentajes de respuestas bien, mal y en blanco por sexo, de cada uno de los bloques que componen la prueba y del total, porque de toda prueba de matemáticas el análisis de las respuestas es necesario como parte del proceso de enseñanza-aprendizaje.

De las 6090 (35x174) respuestas posibles, se contestan 3628 bien, 1038 mal y 1424 no se contestan.

En la tabla 7 se resume en porcentaje las respuestas bien, mal y en blanco por cada uno de los bloques de contenido y por sexo.

Tabla 7.

Porcentajes de respuestas bien, mal y en blanco, por sexo

\begin{tabular}{llllll}
\hline BIEN & NUM & MED & GEO & INC & PCM \\
\hline Hombre & 64.49 & 64.57 & 60.85 & 74.39 & 65.66 \\
Mujer & 60.10 & 54.54 & 49.48 & 63.40 & 56.91 \\
Total & 61.44 & 57.60 & 52.95 & 66.75 & 59.57 \\
\hline MAL & & & & & \\
\hline Hombre & 13.38 & 22.64 & 13.92 & 8.63 & 14.93 \\
Mujer & 15.48 & 26.08 & 18.08 & 11.33 & 17.97 \\
Total & 14.84 & 25.03 & 16.81 & 10.51 & 17.05 \\
\hline BLANCO & & & & & \\
\hline Hombre & 22.13 & 12.79 & 25.23 & 16.98 & 19.41 \\
Mujer & 22.42 & 19.38 & 32.44 & 25.27 & 25.12 \\
Total & 23.72 & 17.37 & 30.24 & 22.74 & 23.38 \\
\hline
\end{tabular}


- De cada diez respuestas, 6 se contestan bien, 2 se contestan mal y 2 se dejan en blanco.

- El bloque de Incertidumbre tiene el porcentaje más alto de respuestas correctas, dos de cada tres estudiantes y el más bajo el de Geometría, uno de cada dos estudiantes.

- De las respuestas mal, el porcentaje más alto corresponde a Medida con uno de cada cuatro estudiantes y el más bajo a Incertidumbre con uno de cada diez estudiantes.

- De las respuestas en blanco Geometría y Medida tienen los porcentajes más alto y más bajo, respectivamente.

La totalidad de los futuros maestros participantes comete errores en alguna de las 35 cuestiones. Se toman los resultados de los diez alumnos con calificaciones de sobresaliente, que tienen 32, 33 o 34 puntos. Hay 4 alumnos con 32 puntos, 1 con 33 y 5 con 34. De ellos 3 son hombres y 7 mujeres. Por curso 1 es de $2 .^{\circ}, 5$ de $3 .^{\circ}$ y 4 de $4 .^{\circ}$.

Se han analizado las respuestas de los quince estudiantes que no llegan a 3 puntos sobre 10 en la prueba, lo que supone tener bien 10 como máximo de las 35 cuestiones de la prueba. Hay 1 alumno con 4,1 con 5,1 con 6,1 con 8,3 con 9 y 8 con 10 . De ellos 1 es hombre y 14 son mujeres. Por curso 10 son de $2 .^{\circ}, 3$ de $3 .^{\circ}$ y 2 de $4 .^{\circ}$.

Hay errores de concepto y de procedimiento, errores graves y errores por falta de conocimientos anteriores, hay errores por una falta de comprensión lectora y otros por "señalar algo" aunque no tenga sentido. Errores que como dicen Socas, Hernández y Palarea (2014) van desde falta de comprensión del texto, a no saber el significado de las palabras y a no comprender globalmente el texto, a errores en operaciones, a falta de conocimientos de los procesos y de los razonamientos.

Algunas respuestas equivocadas se reproducen indicando sexo, edad, curso, resultado de la prueba (PCM), ansiedad (ANS) y actitud (ACT) del alumno, junto con el indicativo del Proceso Cognitivo del ítem ( $R R=$ Razonar y reflexionar, $A A=A$ plicar y analizar, $C R=$ Conocer y reproducir), Dificultad del ítem y Bloque al que pertenece (NUM=Números, MED=Medida, GEO=Geometría, INC=Incertidumbre).

- Mujer, 19 años. Curso $3 .^{\circ}, \mathrm{PCM}=25, \mathrm{ANS}=2.333, \mathrm{ACT}=2.76$. RR-Baja-INC.

4. Pablo y tú vais a jugar lanzando las cuatro monedas al aire. Pablo apuesta por que salgan todas caras. Tú apuestas por que salgan todas cruces.

5CMO4 Elige la afirmación correcta.

A. Pablo tiene más probabilidad de ganar que tú.

B. Tú tienes más probabilidad de ganar que Pablo.

C. Los dos tenéis la misma probabilidad de ganar.

(D.) Seguro que alguno de los dos ganará. 
- Mujer, 21 años. Curso $4 .^{\circ}, \mathrm{PCM}=25, \mathrm{ANS}=1.417 ; \mathrm{ACT}=4.08$. AA-Media-MED.

\begin{tabular}{|l|c|l|l|}
\hline Salida del sol & $6: 50 \mathrm{~h}$ & $5: 13 \mathrm{~h}$ & Pleamar \\
\hline Horas de sol & 15 horas & $11: 24 \mathrm{~h}$ & Bajamar \\
\hline & & $17: 30 \mathrm{~h}$ & Pleamar \\
\hline & & $23: 35 \mathrm{~h}$ & Bajamar \\
\hline
\end{tabular}

6. Luis nunca ha visto una puesta de sol desde la playa e insiste a sus padres para ver si se pueden quedar hasta esa hora. Rellena con números la hora a la que se 6 CM11 pondrá el sol.

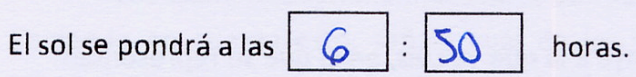

- Mujer, 19 años. Curso 2. ${ }^{\circ}, \mathrm{PCM}=10, \mathrm{ANS}=2.333, \mathrm{ACT}=3.48$. RR-Alta-NUM.

7. Luis quiere calcular la proporción de horas de sol que habrá el sábado. Expresa el resultado en una fracción irreducible y el porcentaje equivalente.

iCM12 Rellena con cifras:
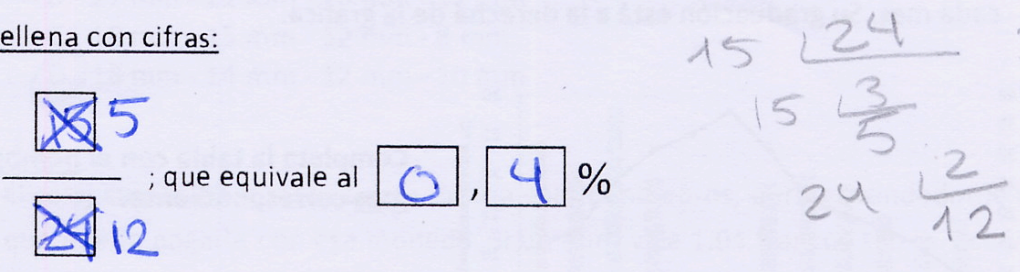

- Mujer, 20 años. Curso $3 .{ }^{\circ} . P C M=23 . A N S=2.167, A C T=3.48$. RR-Alta-NUM.

7. Luis quiere calcular la proporción de horas de sol que habrá el sábado. Expresa el resultado en una fracción irreducible y el porcentaje equivalente.

$6 \mathrm{CM} 12$

Rellena con cifras:
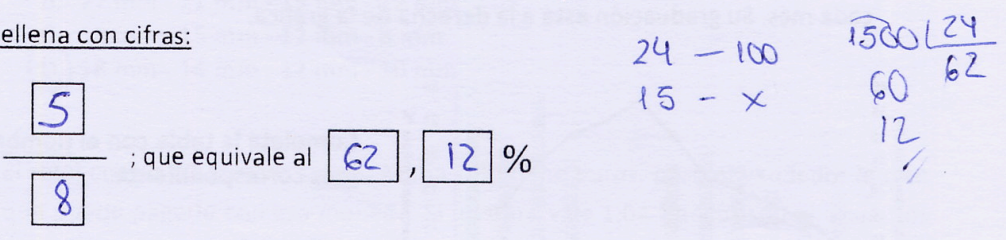

- Mujer, 19 años. Curso 2. ${ }^{\circ}, \mathrm{PCM}=9$. ANS=2.333, ACT=3.64. RR-Media-GEO.

9. Por la tarde empezó a soplar viento del norte y Luis decidió volar su cometa. CM13

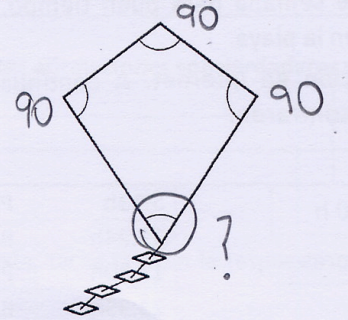

¿Cuánto suman los cuatro ángulos del cuadrilátero que la forman?
A. $180^{\circ}$
B. $270^{\circ}$
C. $360^{\circ}$
D. No tengo suficiente información. 
- Mujer, 19 años. Curso 2. $.^{\circ} \mathrm{PCM}=10 . \mathrm{ANS}=2.333 . \mathrm{ACT}=3.48 . \mathrm{CR}-\mathrm{Media}-\mathrm{NUM}$.

14. Las calles de la piscina están separadas por boyas unidas con cintas. Las boyas tienen que estar a la misma distancia unas de otras. Disponen de cintas de $10 \mathrm{~m}$ y $6 \mathrm{CM} 18$ de $15 \mathrm{~m}$ que tienen que cortar en trozos de la misma longitud. Si han comprado la cantidad justa de cinta y no pueden desperdiciar nada, la longitud máxima de cada trozo de cinta, en metros, es:

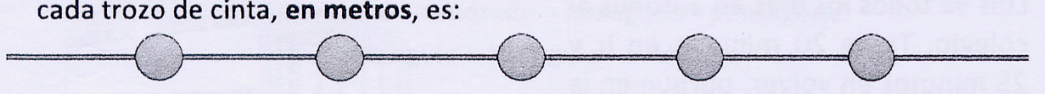
A. 10
B. 5
(c.) 25
D. 3

- Hombre, 21 años,. Curso 2. ${ }^{\circ}, \mathrm{PCM}=23, \mathrm{ANS}=2.833, \mathrm{ACT}=2.8$. AA-Media-MED.

18. El reloj cuesta 80 francos suizos. Maria solo tiene euros, pero el vendedor le dice que puede pagarie con esa moneda. Si un euro vale 1,04 francos suizos, ¿cuántos euros le cuesta a Maria el reloj? Redondea el resultado a céntimos de euro porque no tenemos unidad monetaria más pequeña.
A. $76,92 €$
B. $76,00 €$
C. $83,20 €$
D. $76,95 €$

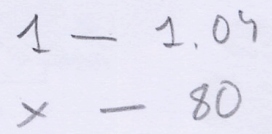
$\times$

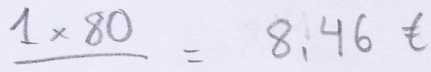
1,04

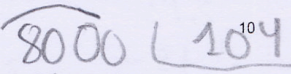
$0480 \quad 8.46$
0640

- Mujer, 22 años. Curso $4 .^{\circ} \mathrm{PCM}=29, \mathrm{ANS}=2.25 ; \mathrm{ACT}=3.84$. AA-Media-MED.

18. El reloj cuesta 80 francos suizos. María solo tiene euros, pero el vendedor le dice 6CM23 que puede pagarle con esa moneda. Si un euro vale 1,04 francos suizos, ¿cuántos euros le cuesta a María el reloj? Redondea el resultado a céntimos de euro porque no tenemos unidad monetaria más pequeña.
A. $76,92 €$
1.04
B. $76,00 €$
$\frac{80}{120}$
800011.04,
C. $83,20 €$
002007
D. $76,95 €$

- Mujer, 21 años. Curso $4 .^{\circ} . P C M=25, A N S=3.667, A C T=2.72$. AA-Media-MED.

18. El reloj cuesta 80 francos suizos. María solo tiene euros, pero el vendedor le dice que puede pagarle con esa moneda. Si un euro vale 1,04 francos suizos, ¿cuántos euros le cuesta a María el reloj? Redondea el resultado a céntimos de euro porque no tenemos unidad monetaria más pequeña.
A. $76,92 €$
B. $76,00 €$
C. $83,20 €$
(D.) $76,95 €$

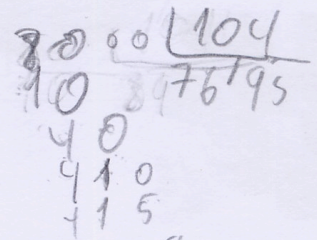


- Mujer, 19 años. Curso 2. ${ }^{\circ}, \mathrm{PCM}=12, \mathrm{ANS}=3.083, \mathrm{ACT}=2.8$. CR-Baja-GEO.

24. Durante el trayecto, Luis se fija en algunas señales de tráfico que aparecen en la carretera. Escribe la figura plana que representa cada imagen, eligiéndola de la 6CM32 siguiente lista:

círculo - cono - cuadrado - hexágono - pentágono rectángulo - triángulo - octógono

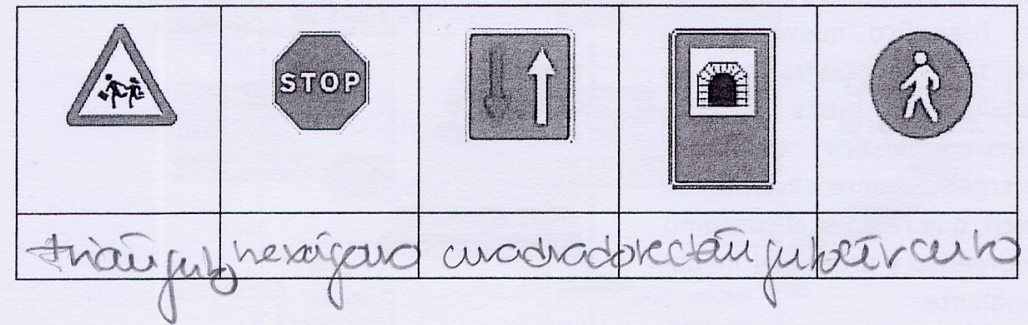

- Mujer, 19 años. Curso $3 .^{\circ}, \mathrm{PCM}=25, \mathrm{ANS}=2.333, \mathrm{ACT}=2.76$. AA-Baja-MED.

25. En la primera clase, la profesora les propone la siguiente actividad

6См33 - En grupos de 4, utilizaréis una cinta métrica para hallar vuestra altura. Después tendréis que ordenar vuestros nombres, de menor a mayor altura.

El grupo de Luis recogió los siguientes resultados:

Luis: $148 \mathrm{~cm}=1,48 \mathrm{~m}$

Marta: $1,4 \mathrm{~m}=1,4 \mathrm{~m}$

Javier: $16 \mathrm{dm}=1,6 \mathrm{~m}$

$m$ din cm mm

Laura: $1,52 \mathrm{~m}=1,52$

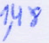

Más alto

Más bajo

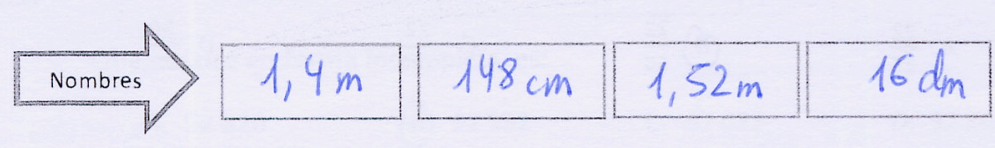

\section{Discusión y conclusiones}

En la Prueba de Competencia Matemática (PCM) se presentan 10 ítems de Proceso Cognitivo "Razonar y Reflexionar" (RR), 15 de "Aplicar y Analizar" (AA) y 10 de "Conocer y Reproducir" (CR) y en los resultados por ítems hay 9 suspensos que corresponde 5 a RR, 3 a $A A$ y 1 a CR, lo que indica que el $50 \%$ de los ítems propuestos de RR se suspenden, mientras que de $A A$ es el $20 \%$ y de $C R$ es el $10 \%$. Pero "utilizar procesos de razonamiento y estrategias de resolución de problemas, realizando los cálculos necesarios y comprobando las soluciones obtenidas" (Real Decreto 126/2014, p. 19387) es fundamental en el currículo básico de Primaria y como dicen Alguacil et al. (2016) "la finalidad principal del profesor en el aula es ayudar a sus alumnos a desarrollar el razonamiento matemático..." (p. 427) y en la prueba el $50 \%$ de los ítems de razonar y reflexionar no se aprueban. 
Al igual que en el estudio de Abrate et al. (2006) la totalidad de los estudiantes que participan en la investigación comete algún error. De cada diez respuestas seis se contestan bien, dos con error y dos se dejan en blanco.

Uno de cada tres futuros maestros no alcanza la Competencia Matemática de $6 .^{\circ}$ de Primaria y en $2 .^{\circ}$ dos de cada cinco, suspende. En $2 .^{\circ}$, por bloques, en Geometría uno de cada dos alumnos suspende y uno de cada cinco suspende los cuatro bloques, mientras que en $4 .^{\circ}$ uno de cada dos estudiantes aprueba los cuatro bloques. En ninguno de los bloques se alcanza una media de Notable y por sexo de cada cinco estudiantes que aprueban tres son hombres y dos son mujeres. El peor bloque es el de Geometría. Peores resultados se dan en $2 .^{\circ}$ (suspenso en Geometría) y mejores resultados en $4 .^{\circ}$ (notable en Incertidumbre). Por sexo mejores resultados en hombres que en mujeres.

La media de la Prueba de Competencia Matemática es de 5.97, por curso es 5.35 en 2. ${ }^{\circ}, 6.32$ en $3 .^{\circ}$ y 6.21 en $4 .^{\circ}$, y por sexo es 6,61 para hombres y 5,69 para mujeres. Resultados mejores que en Nortes y Nortes (2016) en donde la media de la prueba es de 3.55, por curso en $2 .^{\circ}$ es de 3.17 , en $3 .^{\circ}$ de 3.45 y en $4 .^{\circ}$ de 4.11 , y por sexo 3.97 en hombres y 3.37 en mujeres. Las dos pruebas son de contenidos matemáticos de $6 .^{\circ}$ de Primaria, pero la Prueba de Ingreso al Cuerpo de Maestros es de un grado de dificultad superior y en donde solo la mitad de los alumnos contestó bien a la tercera parte de las cuestiones de Matemáticas.

El que uno de cada tres futuros maestros no logre la Competencia Matemática (suspende la Prueba), que en $2 .^{\circ}$ no la logre el $44 \%$, en $3 .^{\circ}$ el $25 \%$ y en $4 .^{\circ}$ el $35 \%$ es un mal dato para el sistema educativo y en particular para la futura docencia en Educación Primaria.

Estamos en la línea de Abrate et al. (2006) de que es más fácil aprender conocimientos nuevos que desaparecer errores viejos, que es mejor explicar el por qué de los errores antes que indicar el modo correcto de hacer las cosas y que es mejor ver el error como instrumento de investigación de los problemas del currículo que como sanción. Es en bloque de Geometría donde uno de cada cuatro alumnos comete error en su respuesta.

Socas et al. (2014) manifiestan que el origen de las dificultades depende del problema, pero también encuentran dificultades vinculadas con la afectividad y las emociones, en determinar el orden en que hay que realizar las operaciones o en identificar con claridad lo que se pide. Muestra de ello son los ejemplos presentados.

Dice Auzmendi (1992) que "si la persona considera que la materia es necesaria y, además, le gusta es lógico que esté más motivada hacia su estudio y utilización" (p. 93). Obtenida la actitud de los futuros maestros se ve que es ligeramente positiva y la ansiedad ligeramente por debajo del valor central. Pero calculada la actitud media de los diez alumnos de sobresaliente (3.504) y la de los quince alumnos con peor nota (3.395) en ambos casos están por encima de la actitud media (3.223) lo que nos indica que a mayor actitud hacia las matemáticas se obtienen mejores resultados en la materia no siempre se realiza, corroborando la baja correlación existente entre prueba y actitud $(r=.202)$ que no llega a ser significativa.

La ansiedad hacia las matemáticas con un valor próximo al central tiene en estudiantes de sobresaliente (2.508) un valor inferior al de los estudiantes con peores notas (2.972), pero en ambos casos inferior a la ansiedad media del estudio (2.985), lo que nos indica que no siempre los alumnos con peores calificaciones tienen mayor ansiedad que la media de alumnos participantes.

La correlación entre Prueba y Ansiedad ( $r=-.265)$ es negativa, baja y significativa, mientras que la correlación entre Actitud y Ansiedad $(r=-.715)$ es negativa, alta y significativa, lo que 
nos indica que a medida que la Ansiedad es mayor la Actitud hacia las Matemáticas es menor.

Es alentador observar como los alumnos de $3 .^{\circ}$, tras cursar 12 créditos de Matemáticas y su didáctica I mejoran los resultados en todos los bloques, pero es preocupante ver que empeoran en $4 .^{\circ}$ tras cursar 9 créditos de Matemáticas y su didáctica II.

Los maestros en ejercicio saben de la necesidad de conocer las matemáticas elementales y el dominio de los contenidos es fundamental, porque no se puede enseñar lo que no se sabe y la respuesta dada por los maestros entrevistados en Martín del Pozo et al. (2013) es concluyente, ya que cuatro de cada cinco maestros entrevistados consideró fundamental dominar los contenidos del área que imparten y el quinto no lo considera fundamental, porque se trata de contenidos muy elementales.

Si tener la Competencia Matemática correspondiente a $6 .^{\circ}$ de Primaria es lograr un "bien", tener una actitud hacia las matemáticas ligeramente positiva (3.2) y una ansiedad neutra (3), entonces se están formando adecuadamente a los futuros maestros, pero si se quiere mejorar la profesionalización de los alumnos del Grado de Maestro de Primaria habrá que mejorar en la docencia del Área de Didáctica de las Matemáticas y recordar a Lacasa y Rodríguez (2013) que son concluyentes en su estudio sobre futuros maestros indicando que quienes acaban dominando mejor la Didáctica de las Matemáticas son aquellos que mejor domina las Matemáticas, y en este estudio la mitad de los ítems de razonar y reflexionar se suspenden.

\section{Referencias}

Abrate, R., Pochulu, M. y Vargas, J. M. (2006). Errores y dificultades en Matemáticas. Análisis de causas y sugerencias de trabajo. Córdoba (Argentina): Universidad Nacional de Villa María. Recuperado de http://unvm.galeon.com/Libro1.pdf

Alguacil, M., Boqué, M. C. y Pañellas, M. (2016). Dificultades en conceptos matemáticos básicos de los estudiantes para maestro. INFAD Revista de Psicología, 1(1), 419-430.

Auzmendi, E. (1992). Las actitudes hacia la Matemática-Estadística en las enseñanzas medias y Universitaria. Bilbao: Mensajero.

Castro, A., Mengual, E., Prat, M., Albarracín, L. y Gorgorió, N. (2014). Conocimiento matemático fundamental para el Grado de Educación Primaria. Inicio de una línea de investigación. En M. T. González, M. Codes, D. Arnau y T. Ortega (Eds.), Investigación en Educación Matemática XVIII (pp. 227-236). Salamanca: SEIEM.

Estrada, A. y Díaz-Palomar, J. (2011). Las actitudes hacia las matemáticas. Análisis descriptivo de un estudio de caso exploratorio centrado en la Educación Matemática de familias. Revista de Investigación en Educación, 9(2), 116-132.

Fennema, E. y Sherman, J. A. (1976). Fennema-Sherman mathematics attitude scales instruments designed to measure attitudes toward the learning of mathematics by males and females. JSAS Catalog of Selected Documents of Psychology, 6(1), 31.

Gómez-Chacón, I. (2009). Actitudes matemáticas: propuestas para la transición del bachillerato a la universidad. Educación Matemática, 21(3), 5-32.

INEE (2016). Evaluación final de Educación Primaria. Competencia Matemática. $6 .^{\circ}$ curso de Educación Primaria. Curso 2015-2016. Recuperado de http://www.mecd.gob.es/dctm/inee/evaluacionfinalprimaria/pruebasinee201617/cm 20166epguia.pdf?documentld=0901e72b8218e044 
Lacasa, J. M. y Rodríguez, J. C. (2013). Diversidad de centros, conocimientos matemáticos y actitudes hacia la enseñanza de las matemáticas de los futuros maestros en España. En TEDS-M Estudio Internacional sobre la formación inicial en Matemáticas de los maestros. IEA. Informe español. Volumen II. Análisis secundario. Madrid-MECD, pp 6597. Recuperado de http://www.mecd.gob.es/dctm/inee/internacional/teds-m-vol2linea.pdf?documentld=0901e72b8171fgcf

Martín del Pozo, R., Fernández-Lozano, P., González-Ballesteros, M. y de Juana, A. (2013). El dominio de los contenidos escolares: competencia profesional y formación inicial de maestros. Revista de Educación, 360, 363-287.

Nortes, R. (2011). Prueba de conocimientos matemáticos de $6 .^{\circ}$ de Educación Primaria: una aplicación a futuros maestros. Investigación e Innovación en Educación Infantil y Primaria (pp. 527-538). Murcia: Universidad de Murcia.

Nortes, R. y Nortes, A. (2016). Resolución de problemas, errores y dificultades en el Grado de Maestro de Primaria. Revista de Investigación Educativa, 34(1), 103-117.

Orden $\mathrm{ECl} / 3857 / 2007$ de 27 de diciembre por la que se establecen los requisitos para la verificación de los títulos universitarios oficiales que habiliten para el ejercicio de la profesión de Maestro en Educación Primaria. Boletín Oficial del Estado. Madrid, 29 de diciembre de 2007, núm. 312, pp. 53747-53750.

Pañellas, M. (2016). Reaprender los conocimientos matemáticos básicos. Tribuna d'educació. Recuperado de http://www.tribunaeducacio.cat/reaprender-losconocimientos-matematicos-basicos/

Real Decreto 126/2014, de 28 de febrero, por el que se establece el currículo básico de la Educación Primaria. Boletín Oficial del Estado. Madrid, 1 de marzo de 2014, núm. 52, pp. 19349-19420.

Rico, L., Marín, A. Lupiáñez, J. L. y Gómez, P. (2008). Planificación de las matemáticas escolares en secundaria. El caso de los Números Naturales. Suma, 58, 7-23.

Segovia, I. y Rico, L. (2011). Matemáticas para maestros en Educación Primaria. Madrid: Pirámide.

Socas, M. M., Hernández, J. y Palarea, M. M. (2014). Dificultades en la resolución de problemas matemáticos para profesores de educación primaria y secundaria. En J. L. González, J. A. Fernández-Plaza, E. Castro-Rodríguez, M. T. Sánchez-Compaña, C. Fernández, J. L. Lupiáñez y L. Puig (Eds.), Investigaciones en Pensamiento Numérico y Algebráico e Historia de la Matemática y Educación Matemática 2014 (pp. 145-154). Málaga: Dpto. de Didáctica de las Matemáticas, de las Ciencias Sociales y de las Ciencias Experimentales y SEIEM. Recuperado de http://www.seiem.es/docs/grupos/pna/ActasPNA2014.pdf 